\title{
Dysplasia epiphysealis hemimelica of the talus
}

\author{
Roberto Azzoni
}

Received: 21 July 2008/Accepted: 14 September 2008/Published online: 23 October 2008

(C) Springer-Verlag 2008

\begin{abstract}
Dysplasia epiphysealis hemimelica is a rare developmental disorder with unknown etiology affecting epiphysis in childhood. The lesion is an osteochondroma arising from the epiphysis and increasing in size until skeletal maturity is reached. Surgical treatment is mandatory when symptoms such as pain, joint impingement or deformation are present, and yields good results when the mass is juxtaarticular or extraarticular. In those cases where articular symptoms are not present and only mass evolution is observed, surgical treatment is not recommended before skeletal maturity has been reached. A case of DEH located in the talus and successfully treated with surgery is presented.
\end{abstract}

Keywords Bone dysplasia - Talus · Trevor's disease

\section{Introduction}

Dysplasia epiphysealis hemimelica (DEH), or Trevor's disease, is a rare developmental disorder affecting epiphysis in childhood. The lesion is an osteochondroma arising from the epiphysis and is typically hemimelic [3].

The term "hemimelica" was coined by Fairbank in 1956 [5]; it derives from two Greek words: "hemi," meaning "half," and "melos," meaning "limb." Fairbank defined DEH as a developmental disorder which is confined to the medial or lateral half of an epiphysis of a single limb.

R. Azzoni $(\bowtie)$

Orthopaedic Department, State University of Milan, Milan, Italy

e-mail: roberto.azzoni@unimi.it

R. Azzoni

Policlinico San Donato, Via Morandi, 30, 20095 San Donato m. (Milan), Italy
The first report of the disorder was written by Mouchet and Belot in 1926 [10], but it was first reviewed by Trevor in 1950 [14] and later by Fairbank in 1956 [5].

\section{Case report}

An eight-year-old male patient presented for examination one year ago with pain and swelling on the medial aspect of the right foot. The swelling had developed since infancy and had gradually increased in size, especially during the year prior to examination. Initially the swelling was painless, but in the year prior to examination it became painful and was aggravated by the use of footwear; there was impingement between the swollen area and the upper side of the footwear. This impingement caused skin inflammation. Additional trauma on this side (ipsilateral) caused more symptoms. No other swelling was found.

During the examination, the size of the swelling measured at the medial foot was $4.5 \times 2.5 \mathrm{~cm}$. The patient had associated bilateral flatfoot. The motility of the ankle was reduced as follows: medial and lateral rotation $25^{\circ}-0^{\circ}-15^{\circ}$; flexion-extension and abduction-adduction were normal. There was no wasting or limb length discrepancy.

At first differential diagnosis was performed between calcified hematoma, os tibialis externum and osteochondroma in DEH. X-ray and MRI exams were performed. Xray (Fig. 1) described the presence of an oval bone tumor measuring $2.4 \times 1.3 \mathrm{~cm}$, with an irregular and thickened structure. No joint deformity or articular surface alterations were described.

MRI (Fig. 2) described an oval bone tumor (measuring $2.5 \times 1.2 \mathrm{~cm}$ ) with anterior and medial peduncle in talus. No joint deformity or articular surface alterations were described. 


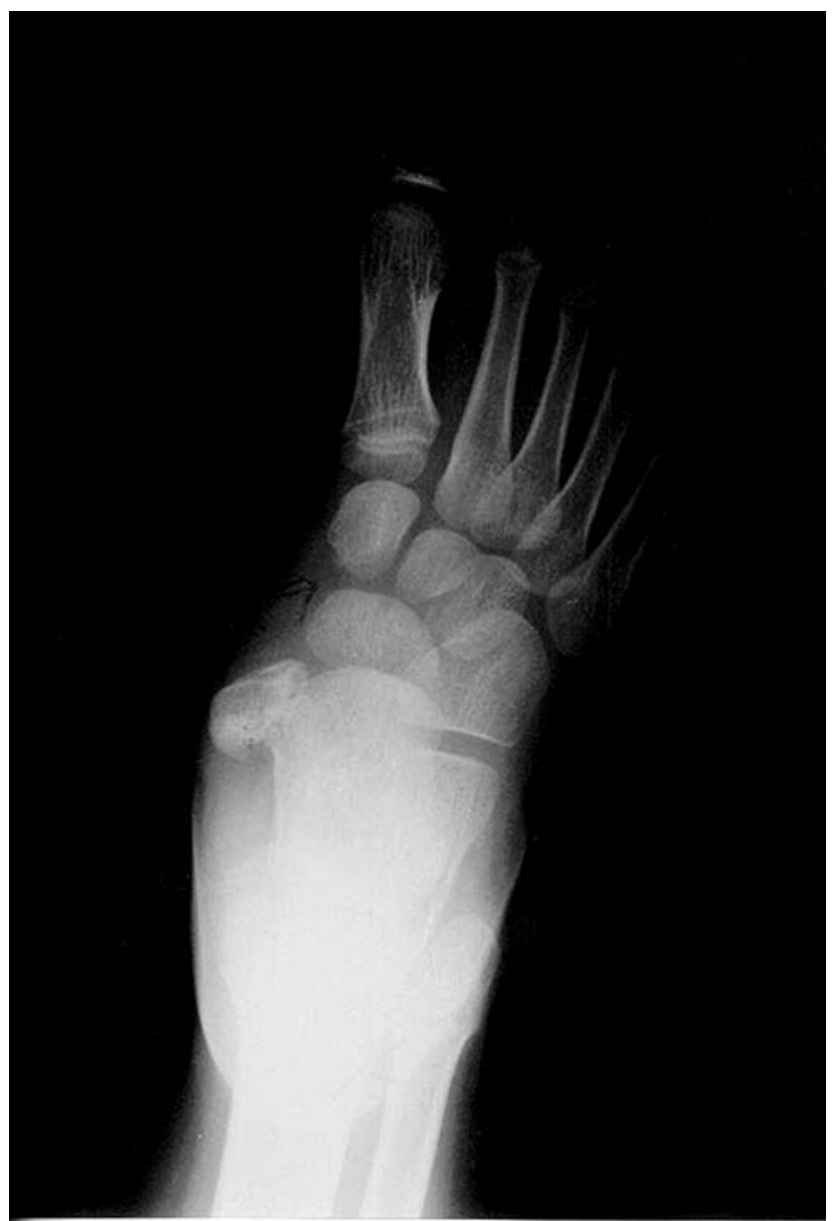

Fig. 1 X-ray exam of the foot with exostosis of the talus

Surgical treatment was performed because the patient presented pain and impingement with footwear. The tumor (Fig. 3) was located on the medial side of the talus, was juxtaarticular, and was "localized" according to the classification of Azouz et al. [2], measuring $2.9 \times 1.9 \mathrm{~cm}$. A large peduncle was present on the medial surface of the talus. The tumor had developed in front of the medial surface of the talus and under it.

Histological exam revealed a benign osteochondroma (Fig. 4). A follow-up examination was performed a year later, and revealed the absence of pain, footwear problems or any other symptoms, as well as a normal motility of the ankle.

The patient's parents provided consent for the publication of the above case report.

\section{Discussion}

The natural history of DEH is a continuous increase in the size of the lesion until skeletal maturity. The frequency of DEH is 1:1,000,000. Approximately 250 cases of DEH

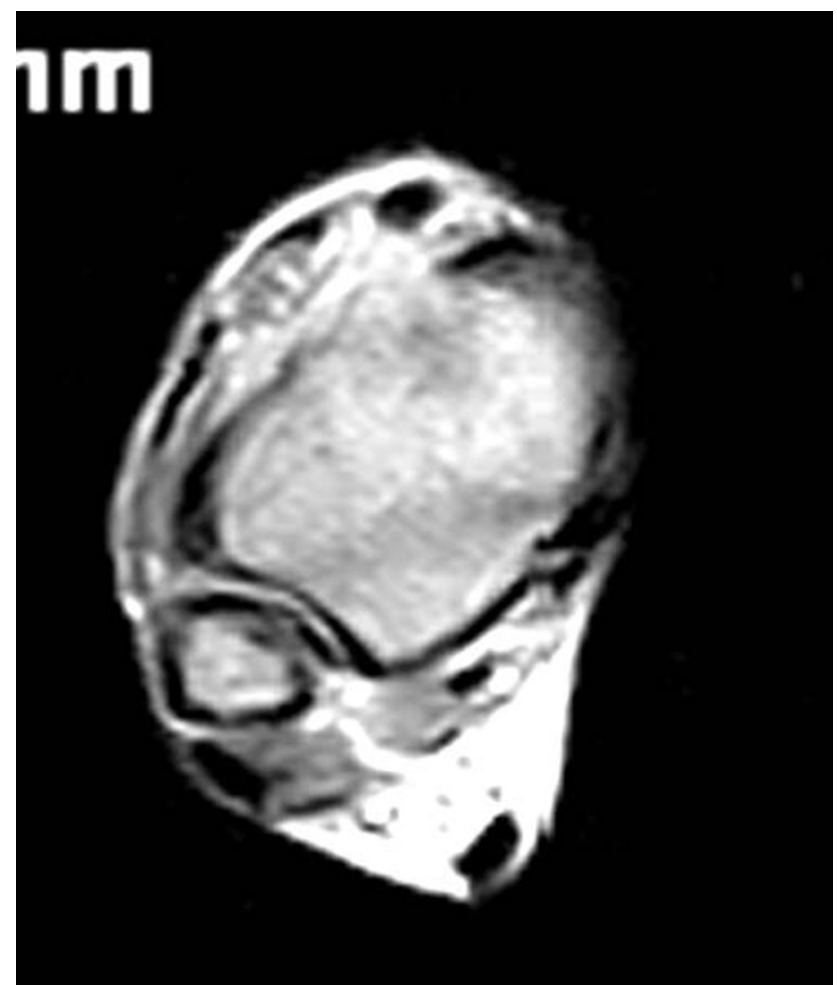

Fig. 2 MRI of the talus confirmed the presence of exostosis of the talus, as well as issues/complications associated with it

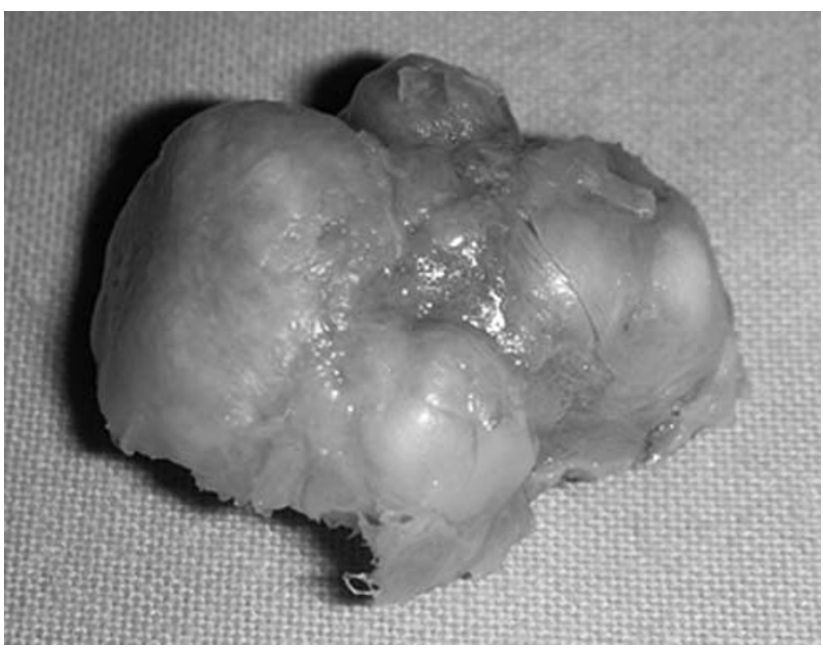

Fig. 3 The removed exostosis

have been documented in the medical literature since 1957. It is probable that a large number of cases have not been properly diagnosed or have simply been diagnosed as osteochondromas $[7,8,12]$. The age of diagnosis is $2-14$; it is rare in adult patients [6]. It is most commonly found in males, with a male-to-female ratio of 3:1 [9].

Based on frequency, DEH occurs most commonly around the knee (both femur and tibia), the talus, and the tarsal navicular and first cuneiform joints. Rare cases have been 


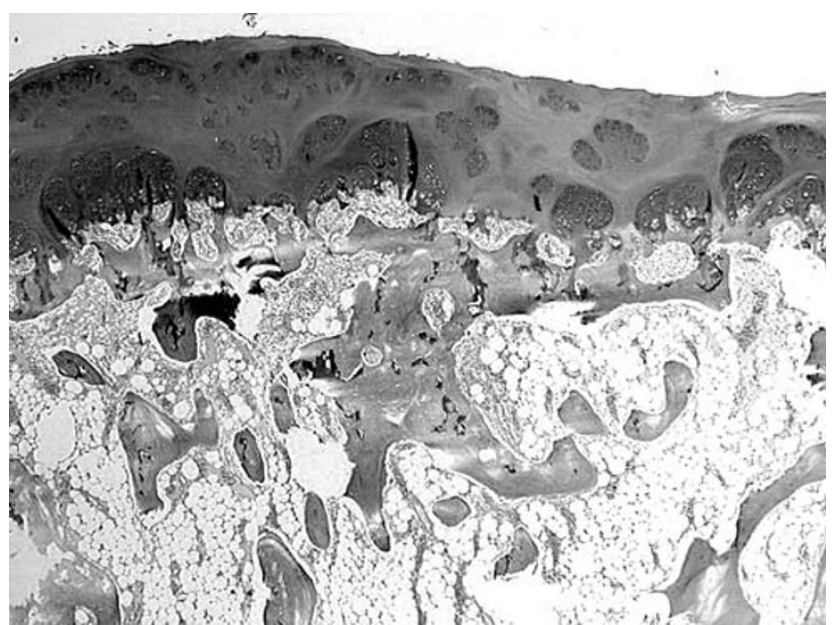

Fig. 4 Histological exam showing the appearance of the exostosis

documented in areas such as the pelvis, patella, calcaneus, cuboid, metatarsal, and phalangeal bones $[1,11]$. The medial side of the epiphysis is most commonly affected.

The etiology of DEH is unknown. Genetic transmission is not a factor. Trevor [14] formulated the hypothesis that the disease is congenital and is related to a damage during the formation of the lower limb bud in early fetal life. This would explain why a preaxial affection would produce the deformity on the medial side of the limb and a postaxial affection on the lateral side [4].

Most cases of DEH are asymptomatic, but they can cause mechanical symptoms depending on their size and location. A painless swelling occurs on the affected joint causing reduced motility, and is sometimes associated with an articular deformity.

The classification of DEH is performed according to Azouz et al. [2] into "localized" (a single bone is affected), "classical" (more than one area in a single lower extremity is affected), and "generalized" types (a whole lower limb from pelvis to foot is affected). Another important classification distinguishes between extra- and intraarticular lesions; we prefer the terms "juxtaarticular" and "articular" lesions (according to Kuo et al. [9]), because most lesions are intracapsular. We can define that extraarticular lesions only occur at extracapsular locations.

The diagnosis is performed by X-ray, CT and MRI exams. Early X-rays show a mass with single or multiple focal ossification centers arising from the affected epiphysis. At later stages, the mass of bone is enlarged and fused with the epiphysis [9]. CT has been recently replaced by MRI. CT assists in defining the anatomic relationship between the mass and its parent bone and helps when evaluating the condition of the articular cartilage and soft tissues [3]. MRI shows the extent of the mass, the joint deformity, and the status of the articular surface, especially on T2-weighted gradient-echo images [9].
Some authors recommend the use of scintigraphy in the final stages of diagnosis [13]. This exam shows a marked increase in the uptake at the location of the mass, comparable to the growth plate of the epiphysis of interest. Scintigraphy is useful because it permits the entire skeleton to be evaluated using radiation doses that are much lower than those needed for a skeletal survey [13].

Treatment of DEH can be surgical in those patients with articular impingement or pain. In those cases where articular symptoms are absent, simply observing the evolution of the mass is the recommended approach $[1,3,4,9,11]$. Histology exams reveal the presence of a benign osteochondroma. Malignant transformation has not been reported [3, 9].

One typical location for DEH is the talus. The current medical literature reports about 30 cases located in the talus, representing $12 \%$ of all cases. In such cases, the primary symptom is a painless lump of gradually increasing size. Mechanical symptoms are frequent, particularly due to articular or footwear impingement. Alterations of the range of motion of the ankle are frequent. Articular deformation is possible when the mass is intraarticular.

Dysplasia epiphysealis hemimelica is still a rare pathology, but the number of documented cases is on the rise. When examining a child with swelling in a medial or lateral location in the proximity of the joint, it is mandatory to consider the possibility of DEH, especially if there is a joint deformation with alterations in the range of motion. Diagnosis is relatively simple using X-ray and MRI exams. Histological exams confirm the diagnosis. It is possible to observe bone mass when there are no symptoms; surgical treatment is mandatory when symptoms like pain, joint impingement or deformation are present. Surgical treatment is successful when the mass is juxtaarticular or extraarticular. When the mass is intraarticular, early surgery may cause secondary osteoarthritis. Therefore, if there are no symptoms and skeletal maturity has not yet been reached, only visual examination is recommended.

Conflict of interest statement The authors declare that they have no conflict of interest related to the publication of this manuscript.

\section{References}

1. Arkader A, Friedman JE, Moroz L, Dormans JP (2007) Acetabular dysplasia with hip subluxation in Trevor's disease of the hip. Clin Orthop Relat Res 457:247-252

2. Azouz RM, Slomic AM, Marton D, Rigault P, Finidori G (1985) The variable manifestations of dysplasia epiphysealis hemimelica. Pediatr Radiol 15:44-49

3. Bakarman K, Letts RM (2006) Displasia epiphysealis hemimelica (online article). http://www.emedicine.com/orthoped/topic628.htm. Last accessed 22 Sept 2008 
4. Boshale SK, Dholakia DB, Sheth BA, Srivastava SK (2005) Dysplasia epiphysealis hemimelica of the talus: two case reports. J Orthop Surg 13(1):79-82

5. Fairbank TJ (1956) Dysplasia epiphysealis hemimelica (tarsoepiphyseal acalasis). J Bone Joint Surg Br 38:237-257

6. Freihaut RB, O'Keane JC, Stephens MM (2007) Dysplasia epiphysealis hemimelica with associated osteochondromal lesion of the talus: a case report and review of the literature. Foot Ankle Int 28(6):727-730

7. Joshi D, Kumar N, Singh D, Lal Y, Singh AK (2005) Osteochondroma of the talus in a male adolescent. J Am Podiatr Med Assoc 95(5):494-496

8. Keser S, Bayar A (2005) Osteochondroma of the talar neck. J Am Podiatr Med Assoc 95(3):295-297

9. Kuo RS, Bellemore MC, Monsell FB, Frawley K, Kozlowsky K, Doc F (1998) Dysplasia epiphysealis hemimelica: clinical features and management. J Pediatr Orthop 18(4):543-548
10. Mouchet AA, Belot J (1926) Tarsomegalie. J Radiol Electrol 10:289-293

11. Segal LS, Vrahas MS, Schwentker EP (1996) Dysplasia epiphysealis hemimelica of the sacroiliac joint: a case report. Clin Orthop Relat Res 333:202-207

12. Takeuchi $H$, Ito $K$, Ogino $T$, Hasegawa $T$, Kitamura $T$, Ishii $S$ (2003) A case of osteocartilaginous mass involving the coronoid process of the ulna: solitary osteochondroma or dysplasia epiphysealis hemimelica? J Shoulder Elbow Surg 12(5):510-513

13. Teixeira AB, De Camargo E, Elba C, Santos A, Lima M, Ramos C, Camargo EE (2001) Scintigraphic findings of dysplasia epiphysealis hemimelica: a case report. Clin Nucl Med 26(2):162-165

14. Trevor D (1950) Tarso-epiphysial aclasis: a congenital error of epiphysial development. J Bone Joint Surg Br 32:204-213 